\title{
Perfil docente, bienestar y competencias emocionales para la mejora, calidad e
} innovaciòn de la escuela

\section{Teacher profile, well-being and emotional competencies for the improvement, quality and innovation of the school}

\author{
Ana María Ávila Muñoz ${ }^{* 1}$ \\ *Universidad Autónoma de Madrid.
}

\section{RESUMEN}

Esta investigación muestra la relación existente entre competencia emocional, bienestar personal y estilo educativo, entendiendo estos aspectos como esenciales a la hora de promover procesos de mejora en la escuela, y señalando la importancia de la formación docente en educación emocional. Ya que un profesor emocionalmente competente presentará un mayor bienestar personal y optará por un estilo educativo asertivo.

Gestionar las emociones y el bienestar personal se convierte en un elemento básico para

1 ORCID: $0000-0003-3682-3087$ Maestra funcionaria en la Comunidad de Madrid, psicopedagoga y doctoranda en la Universidad Autónoma de Madrid, ana. avilamunoz@educa.madrid.org, $\quad$ https://orcid.org/00000002-2007-9444

iraida.campos@umcc.cu favorecer el desarrollo integral del alumno, un elemento clave para trabajar con los futuros docentes en su formación inicial (Bisquerra, 2007; López-Goñi y Goñi, 2012) aunque en la práctica ocupa un segundo plano dentro del conjunto de las competencias docentes.

Enesta investigación participaron 230 profesores, completaron cuestionarios sobre competencia emocional, bienestar y estilos educativos. Los resultados muestran, mayoritariamente, que aquellos docentes que tienen alta puntuación en competencia emocional, la adquieren también en bienestar personal y además son aquellos con estilo asertivo.

Todo ello confirma que aquellos docentes con mayor competencia emocional disfrutan igualmente de un mayor bienestar personal, y esto correlaciona con su perfil docente y 
estilo educativo, y por lo tanto en el proceso de enseñanza/aprendizaje y los agentes implicados.

PALABRAS CLAVE: mejora educativa, competencia emocional, bienestar personal, perfil docente, estilo educativo.

\section{ABSTRACT}

This research shows the relationship between Emotional Competence, Well-Being and Educational Style, understanding its importance to promote school improvement processes.

230 participants completed questionnaires of Emotional Competence (Profile of Emotional Competence), Well-Being (Scale of Well-Being) and Teacher Profile (Scale PEC). The results show that the majority of teachers have an assertive educational profile, a high score in Emotional Competence and that there is a very significant relationship between teachers with high score in Emotional Competence and Wellbeing simultaneously.

This fact confirms that more emotionally competent teachers enjoy greater personal wellbeing.

KEYWORDS: educational improvement, emotional competence, well-being, teacher PROFILE, EDUCATIONAL PROFILE.

\section{FUNDAMENTACIÓN TEÓRICA}

Vivimos en una sociedad cambiante, rápida, desenfrenada, todo esto se refleja en los centros educativos y por ende en las aulas.

Hasta hace unas décadas la preocupación sobre el aprendizaje de los discentes se ha centrado en una mejora del rendimiento académico en una potencialización del desarrollo cognitivo del alumno, "cómo poder aprender más en menos tiempo", entendido este aprender como una acumulación de contenidos. La preocupación en esta acumulación, con los avances tecnológicos de los últimos años, ha quedado obsoleto y desnaturalizado. Los docentes se encuentran en un momento de alta demanda en el desarrollo de todas las competencias de sus alumnos, las leyes educativas actuales tanto a nivel nacional como a nivel europeo, destacan una visión del aprendizaje vinculado en su totalidad con el desarrollo de las competencias clave, recogiendo "un saber, un hacer y un sentir". Por lo tanto los docentes deben de poder satisfacer estas demandas a partir de una formación tanto en conocimientos pedagógicos, psicológicos y metodológicos, ya que tienen como finalidad máxima la mejora de la escuela.

La mejora de la escuela debe entenderse no como un objetivo en sí mismo sino como un medio para llegar a un cambio en la sociedad y crear una sociedad más justa a partir de la escuela (Murillo y Hernández-Castilla, 2014). La investigación sobre las emociones favorece un cambio del énfasis de lo cognitivo a lo emocional (Fernández-Abascal, Jiménez y Martín, 2003) para posibilitar el aprendizaje a lo largo de la vida (Gordillo, 2015).

Saarni (1997, 2000) define Competencia Emocional como el conjunto de habilidades, capacidades y actitudes que están estrechamente interrelacionadas con el entorno social. Relaciona la Competencia Emocional con la demostración de autoeficacia al expresar emociones en las transacciones sociales ("emotion-eliciting social transaccions"). Por lo tanto se entiende la Competencia Emocional aplicada en un contexto.

Pérez y Repetto (2007) denominan a su modelo holístico o integrado, en él además de aspectos psicológicos (rasgos de personalidad) intervienen otros de corte fundamentalmente sociológico, político y profesional. El contexto es la función docente y la enseñanza de esta, es decir, la formación del profesorado (López-Goñi I., Goñi-Zabala, J.M., 2012). 
Hué (2008) señala que las competencias emocionales se refieren, de una parte, a las capacidades relativas a la mejora personal como el autoconocimiento, la autoestima, el autocontrol o la motivación, la creatividad, la capacidad para el cambio o para la toma de decisiones; y de otra, a aquellas relativas a las relaciones con los demás como la empatía, la capacidad para establecer una adecuada comunicación para trabajar en equipo, para resolver conflictos, para analizar las necesidades de la sociedad o los mercados, para ser líderes en diferentes situaciones sociales.

Bisquerra (2007), destaca a las competencias emocionales como el conjunto de conocimientos, capacidades, habilidades y actitudes necesarias para comprender, expresar y regular de forma apropiada los fenómenos emocionales $\mathrm{y}$ entiende que pueden agruparse en cinco bloques: conciencia emocional, regulación emocional, autonomía personal, inteligencia interpersonal, habilidades de vida y bienestar.

Las investigaciones realizadas en la actualidad sobre competencia emocional se desarrolla en investigar por un lado análisis y contraste con otras variables, y cómo desarrollarlas en el ámbito educativo (Seiz, J., Vossb, T. y Kuntera, M., 2015), (Dolev, N.,y Leshemb S., 2016), (Martínez, L., 2016), (Carter, D., 2016), (Yildizbas, F., 2017), (Schonert-Reichl K., 2017), y por otro lado en realizar unos instrumentos con una mayor validez y fiabilidad.(Peterson,E. R., Farruggia, S. F., Hamilton, R. J. y Brown, G. T. L.,2013), (Torrente C., Nathanson, L., Rivers, S., Brackett, M. 2015), (Twyman, J., y Redding, S., 2015).

El bienestar personal, subjetivo o psicológico, ha sido definido como "experiencia emocional placentera" (Diener, 1994). Existen dos grandes aproximaciones en la conceptualización del bienestar, la hedónica y la eudaimónica, que se centran en sentirse bien y funcionar bien, las dos se complementan.
Una propuesta integradora es el modelo de bienestar psicológico propuesto por Ryff (1989a) que considera que el bienestar psicológico es un constructo multidimensional que incluye diferentes actitudes vitales tales como: autoaceptación, experimentar relaciones positivas con los otros, autonomía, dominio del entorno, propósito vital, crecimiento personal.

Algunos de los factores a destacar son la implicación en metas y el uso de un afrontamiento efectivo (Diener, Suh, Lucas, y Smith, 1999; Lyubomirsky, King, y Diener, 2005; Ryff, 2014), que tienen un potente efecto sobre el desarrollo de bienestar.

Diferentes investigaciones muestran que la satisfacción con la vida está vinculada con el autoestima, el ajuste escolar, los perfiles motivacionales, los recursos de apoyo y el clima social (Cava \& Musitu, 1999; Ferragut y Fierro, 2012; Furr y Funder, 1998; Huebner, 1991a, 1991b; Lewinsohn et al., 1991; Martínez-Antón et al., 2007; McCullough, Huebner y Laughlin, 2000; Moreno-Murcia y Silveira, 2015; Pacheco y Fernández Berrocal, 2004; Van Aken y Asendorpf, 1997; Ying y Fang-Biao, 2005).

Por lo tanto el trabajo de la gestión de las emociones y del bienestar personal se convierte en un elemento básico parafavorecerel desarrollo integral del alumno. $Y$ que son elementos claves a trabajar con los futuros docentes en su formación inicial tal y como lo destacan algunos autores (Bisquerra, 2007; López-Goñi y Goñi, 2012), aunque en la práctica ocupan un segundo plano dentro del conjunto de las competencias docentes. En este sentido, Gallego y Gallego (2004) señalan que la adecuada gestión emocional y la armonización entre lo cognitivo y lo emocional debe contemplarse plenamente en el currículum. Además, Livia y García (2015) exponen cómo el contexto educativo representa un lugar privilegiado para la promoción de las habilidades y el desarrollo de competencias socioemocionales, las cuales contribuyen de 
forma positiva al bienestar personal y social de las personas.

En esta línea, Bisquerra (2007), afirma que el profesorado emocionalmente competente está mejor preparado para relacionarse de manera positiva y adecuada con la comunidad educativa, aumentando la eficiencia de la educación. En las aulas, cada día surgen infinidad de dificultades a las que hacer frente, como pudieran ser: el absentismo escolar, la falta de motivación e interés por el estudio por parte de los alumnos $y / o$ las inadecuadas relaciones interpersonales entre ellos. Todo ello, como uno fácilmente puede comprender, desemboca en un ambiente nada favorecedor para el aprendizaje. A su vez, la propia situación de los docentes, inmersos en el cumplimiento de normas, pautas y gestiones requeridas por las autoridades, con tiempos y recursos bastantes limitados, pendientes de realizar gestiones y actividades propias de la escuela, así como envueltos en sus propios problemas personales, no ayuda a que la situación inicial mejore. (Hernández, 2017).

La formación inicial y el desarrollo profesional de los maestros están indudablemente relacionados con sus formas de intervención en su práctica educativa. Este es un tema al que no se le ha prestado la suficiente atención, pues tradicionalmente se ha dado énfasis a los métodos, recursos didácticos, técnicas, planes y programas que van actualizándose. A todo ello, debemos sumar las reformas que entran en vigenci+96a continuamente. (Hernández 2017).

Una experiencia de formación en competencias emocionales, Hué (2011) es el estudio, denominado "Habilidades docentes del profesorado e inteligencia emocional", se llevó a cabo con docentes universitarios entre los años 2004 y 2006. La actividad formativa partía del "Proyecto Tuning", en el que establecía una diferencia entre las competencias específicas, relacionadas con la disciplina en la que fue formado el docente, y las genéricas, relacionadas con la gestión de la información, su perfil pedagógico y las competencias emocionales. Esta experiencia nos lleva a reconocer por qué es importante que el rol docente sea redefinido, tomando en cuenta las transformaciones sociales y educativas. La idea sería sustituir el rol tradicional del docente, en el que sólo se destaca la dimensión cognitiva, por otro más profesionalizado, con una visión de formación integral de los alumnos.

López-Goñi y Goñi-Zabala (2012) a partir de un estudio sobre las propuestas formativas que actualmente se hacen desde las instituciones, (analiza Alemania, España, Francia, Inglaterra y Portugal), de que las competencias emocionales ocupan un lugar menor en el conjunto de competencias docentes. Destacan que se debería de destinar algo más de atención a las competencias emocionales de tipo intrapersonal, reflexionar sobre la importancia de los contextos de uso de las competencias profesionales, y buscar un equilibrio entre dichos contextos.

Gordillo (2015) en su tesis doctoral "Análisis de la competencia emocional de los futuros docentes extremeños" en la Universidad de Extremadura; y Torrijos (2016) "Desarrollo y evaluación de competencias emocionales para profesores mediante una intervención por programas" en la Universidad de Salamanca evalúan las competencias emocionales de los futuros docentes:

Los estudios con respecto a la preocupación y relación del bienestar personal con otras variables se pueden ver en las tesis de : Freire (2014) "El bienestar psicológico en los estudiantes universitarios: Operativización del constructo y análisis de su relación con las estrategias de afrontamiento" en la Universidad de A Coruña; Yuste (2015) "Creencias de autoeficacia docente en estudiantes de magisterio: Análisis de su relación con variables de personalidad y bienestar psicológico y estudio del cambio" en la Universidad de Valencia; y 
García-Jiménez (2017) "Bienestar Emocional en Educación: Empecemos por los Maestros” en la Universidad de Murcia.

La relación entre competencia emocional y bienestar ha sido estudiada por diferentes autores y en distintos contextos. Limonero, Tomás-Sábado , Fernández-Castro, GómezRomero ,y Ardilla-Herrero (2012), destacan en su investigación realizada con universitarios, que la regulación emocional ejerce un efecto mediador en la satisfacción con la vida y en la forma de afrontar los acontecimientos estresantes. La capacidad de afrontar situaciones difíciles aumenta, cuando podemos entender las razones que mueven a las personas que las crean, las emociones envueltas en esas situaciones, y los efectos que nuestras acciones tendrán en las otras personas. Todo ello, hace que las personas con inteligencia emocional puedan sufrir menos en situaciones negativas y disfrutar más en las positivas, derivando, por tanto, de esas situaciones, mayor satisfacción que las personas con baja inteligencia emocional. Estos resultados se relacionan con otros estudios con adultos (Pérez-Escoda y Alegre, 2016) y con estudiantes universitarios (Extremera y Rey, 2016a).

Bisquerra y Hernández (2017), crean el programa "Aulas Felices", para alumnos de 3 a 18 años. Se expone como el desarrollo del bienestar debería ser una de las finalidades de la educación, lo cual repercutirá en el profesorado, alumnado, familias y por extensión a la sociedad en general. Argumentan sobre la importancia de potenciar el bienestar en la educación, se presentan actividades prácticas y estrategias de intervención, con especial referencia a la formación del profesorado. EI GROP (Grup de Recerca en Orientación Psicopedagógica) de la Universidad de Barcelona está realizando investigaciones en esta línea.

En la etapa de educación primaria, Ros, Filella, Riber y Perez-Escoda (2017) relacionan en su estudio las variables competencia emocional, clima de aula, bienestar, rendimiento académico y autoestima, concluyendo que la variable que más explicaba el grado de ansiedad fue en todo momento las competencias emocionales. Así pues, se puede afirmar que el desarrollo de las capacidades emocionales es uno de los indicativos más ajustados en cuanto a la predicción del bienestar de los alumnos (Ciarrochi, Heaven, y Supavadeeprasit, 2008). Consistente con esta idea, este estudio señaló que de todas las variables estudiadas, las competencias emocionales mostraron un mayor peso en la predicción de la ansiedad presentada por los alumnos.

Por otro lado, atendiendo a profesionales de la educación como son los orientadores, ÁlvarezRamírez, Pena y Losada (2017), realizan una investigación en la que plantean la mejora del bienestar personal a partir de la inteligencia emocional. Examinan los beneficios de la inteligencia emocional en diversas dimensiones del bienestar personal y laboral tales como satisfacción vital, compromiso en el trabajo (engagement) y percepción de estrés en una muestra de orientadores, dado que las habilidades emocionales han sido identificadas como necesarias en el perfil profesional de los orientadores laborales y educativos.

López-Casa, Pérez-Escoda y Alegre (2018) Analizan la relación entre el nivel de competencia emocional y el bienestar derivado de experiencias en contextos específicos así como el nivel de satisfacción con la vida en adolescentes. Una de sus hipótesis es que la competencia emocional modera la relación entre satisfacción en cada uno de los dominios específicos y la satisfacción con la vida. Los resultados confirmaron esta hipótesis, para la satisfacción con los recursos de afrontamiento, pero no para la satisfacción en otros dominios específicos.

Cada docente cuenta con un estilo educativo específico, Magaz y García Pérez (2011) 
definen estilos educativos como " el conjunto de ideas, creencias, valores, actitudes y hábitos de comportamiento que padres y profesores mantienen respecto a la educación de sus hijos y alumnos".

Todas las personas están inmersas en un medio social y reciben influencias desde su infancia y hasta la madurez tanto de modelos directos como familia y profesores; como de modelos indirectos medios de comunicación y literatura, entre otros. Este proceso constituye el modo de percibir el mundo, y las propias experiencias "modulando" la manera de interactuar con los demás y consigo mismo, es decir, va constituyendo los hábitos de comportamiento. Los estilos educativos son el conjunto de estilos cognitivos y de comportamiento que conforman el "estilo de vida" de los docentes(Magaz y García Pérez, 2011).

Estos estilos son clasificados como: Educación sobreprotectora. Los docentes piensan que hay que proteger al niño evitando que se exponga a situaciones de riesgo en las que pueda sufrir algún perjuicio. Se consideran excesivamente responsables de su desarrollo; Educación inhibicionista. Se parte de la premisa de que cada uno aprende por su cuenta el docente únicamente tiene que posibilitar el desarrollo normal sin poner impedimentos al mismo; Educación punitiva. Se entiende que las personas deben de actuar según las normas y códigos de convivencia, el comportamiento reglado no merece ser recompensado. No se entiende que se cometan fallos; Educación asertiva. El niño tiene que aprender a comportarse como los adultos desean, el aprendizaje de una habilidad requiere disponer de otras habilidades, y se entiende el fallo como parte del aprendizaje.

Investigaciones actuales muestran la influencia del estilo asertivo del docente en la competencia social de sus alumnos y en la cohesión del grupoclase (Villena, M.D., Justicia, F. y Fernández, E., 2016), la asertividad influye positivamente en el desarrollo de la competencia social de los alumnos, aumenta la cohesión entre los miembros del grupo-clase. Se configura, así, como una competencia interpersonal deseable en el perfil profesional docente.

Investigaciones realizadas con profesorado universitario han afirmado el vínculo entre la personalidad del docente y su capacidad para actuar correctamente en el aula, así como la influencia de la competencia social del profesor sobre la del alumnado (Vališová, 2011).Vališová (2011) sostiene la influencia de la competencia social del docente sobre la del alumno en términos de su capacidad para manejar grupos de trabajo, comunicarse y cooperar con el grupo, discutir y negociar de modos no violentos, hacer frente a aspectos emocionales referentes a sí mismo y a los demás, o su habilidad para determinar las reglas que gobiernan las interacciones de los estudiantes en el aula, así como las normas y el respeto recíproco entre ellos. Aspectos, todos ellos, que forman parte de la práctica de cualquier profesor, y que conllevan la creación de un clima de aula favorecedor del desarrollo de capacidades socio-emocionales (Merchán y González, 2012).

La asertividad se configura, por tanto, como una competencia interpersonal deseable en el perfil del buen docente ya que además, como cualquier otra habilidad de interacción social, contribuye al bienestar psicológico de profesores y alumnos así como a un aprendizaje de calidad (Talvio et al., 2013).

\section{OBJETIVO DE LA INVESTIGACIÓN}

Evaluar la competencia emocional, bienestar personal y el estilo educativo.

Analizar la existencia de correlaciones entre las diferentes variables.

\section{MÉTODO}

Participan 230 profesores de los que el $85,8 \%$ 
son mujeres. La edad media es de 40 años, y la experiencia docente media de 14,3 años. Son maestros el $78,8 \%$, profesores de secundaria $18,4 \%$ y el $2,8 \%$ profesores de universidad. Trabajan en centros públicos el $79,2 \%$, centros concertados $15 \%$ y centros privados $5,8 \%$.

Los cuestionarios utilizados son:

PEC (Profile of Emotional Competence. (Brasseur et al. 2013). Adaptación al español (Páez, Puente, Martínez, Ubillos, Filella y Sánchez, 2016). Evalúa la autopercepción de las competencias emocionales intra e interpersonales. Consta de 50 ítems distribuidos en una escala con rango 1-5 en la versión original (total desacuerdo-total acuerdo).

Bienestar Psicológico (Riff, 1995). Adaptación al español (Díaz, Blanco, Moreno-Jiménez, Gallardo, Valle, Dierendonck, 2006). Considera este constructo con base en seis dimensiones: Auto aceptación, relaciones positivas, autonomía, dominio del entorno, propósito en la vida y crecimiento personal. Esta escala está compuesta por 39 ítems a los que los participantes responden utilizando un formato de respuesta tipo likert con puntuaciones comprendidas entre 1 (totalmente en desacuerdo) y 6 (totalmente de acuerdo), para un total de 234 puntos como máximo.

Perfil Estilo Educativo (Magaz, y García-Pérez, 2011), sobre actitudes hacia la educación. El cuestionario tiene un total de 48 frases, correspondientes a ideas, creencias, actitudes y valores de tipo sobreprotector, inhibicionista, punitivo y asertivo, respectivamente. Las frases no están ordenadas y se presentan de manera aleatoria.

Se utilizaron redes sociales y el correo electrónico para recabar los datos y el SPSS para su análisis.

\section{RESULTADOS}

Analizando la tabla 1 se observa que la puntuación en competencia emocional es alta (198) y los estilos educativos mayoritarios son, en primer lugar, el asertivo (66,5\%), estos docentes se sienten tranquilos mientras comprueban los progresos, satisfechos cuando cumplen sus instrucciones y cuando expresan sus gustos y deseos fijando su atención en los progresos, en los esfuerzos y logros. Tienen "paciencia activa", esto quiere decir que confían en el progreso hacia los objetivos conductuales deseables. Mientras se encuentra en las fases intermedias, se muestran comprensivos, aceptan su nivel de imperfección y elogian sus esfuerzos (Magaz, y García-Pérez, 2011) y, en segundo lugar, el falso asertivo $(22,2 \%$,$) , su verdadero estilo es$ punitivo, pero ha respondido al cuestionario con alta deseabilidad social. Se ve a la hora de analizar los datos ya que dan alta puntuación en asertividad y punición.

Con respecto a los resultados de bienestar personal se destaca que siendo los factores que conforman el bienestar: autoaceptación, relaciones positivas, autonomía, dominio del entorno, propósito de vida y crecimiento personal. Los docentes han obtenido un alto índice en bienestar y en todos sus factores, señalando que en el que más puntuación han obtenido es en el de autonomía y en el que menos en el de autoaceptación, aunque la diferencia de puntuación entre factores no es muy significativa. 


\begin{tabular}{l} 
Tabla 1. Media moda, desviación estándar, varianza, mínimo y máximo de los factores del PCE, PBP \\
y PEE. \\
\hline
\end{tabular}

CE.: Competencia emocional (PEC); F.Auto.: Factor autoaceptación (PBP); F. Rel Pos.: Factor relaciones positivas (PBP); F. Aut.: Factor autonomía (PBP); F. Dom. Ent.: Factor dominio del entorno (PBP); F. Prop. Vid: Factor Propósito de vida (PBP); F. Crec. Per: Factor crecimiento personal (PBP); Sobre.: Total sobreprotector (PEE); Aser.: Total asertivo (PEE); Inhi.: Total inhibicionista (PEE); y Puni.: Total punitivo (PEE).

En la tabla 2 se observa claramente que el perfil de estilo educativo mayoritario es asertivo con un porcentaje de $66,5 \%$ seguido del falso asertivo $22,2 \%$ siendo significativa la poca puntuación obtenida por los demás estilos, siendo el menor el estilo sobreprotector.

Tabla 2. Perfil docente del PEE.

\begin{tabular}{|l|l|l|l|}
\hline \multicolumn{2}{|c|}{} & Frecuencia & Porcentaje \\
\hline \multirow{4}{*}{} & Sobreprotecto & 1 &, 4 \\
\cline { 2 - 4 } & Punitivo & 2 &, 9 \\
\cline { 2 - 4 } & Asertivo & 153 & 66,5 \\
\cline { 2 - 4 } & Falso asertivo & 51 & 22,2 \\
\cline { 2 - 4 } & No definido & 2 &, 9 \\
\cline { 2 - 4 } & Inhibicionista & 2 &, 9 \\
\cline { 2 - 4 } & Total & 211 & 91,7 \\
\hline
\end{tabular}

En la última tabla, (la tabla 3 ) se presenta el estudio de correlaciones entre los factores de Perfil de Competencia Emocional, Escala de Bienestar PSicológico y Perfil de Estilo Educativo.

Se destaca alta correlación entre la puntuación de competencia emocional y todos los factores medidos de bienestar psicológico.

Una alta correlación negativa se observa entre el factor autonomía y estilo sobreprotector.

También se observa el resultado de alta correlación entre el estilo de inhibición y sobreprotección.

Tabla 3. Correlaciones entre los factores de Perfil Competencia Emocional, Escala de Bienestar Psicológico y Perfil de Estilo Educativo.

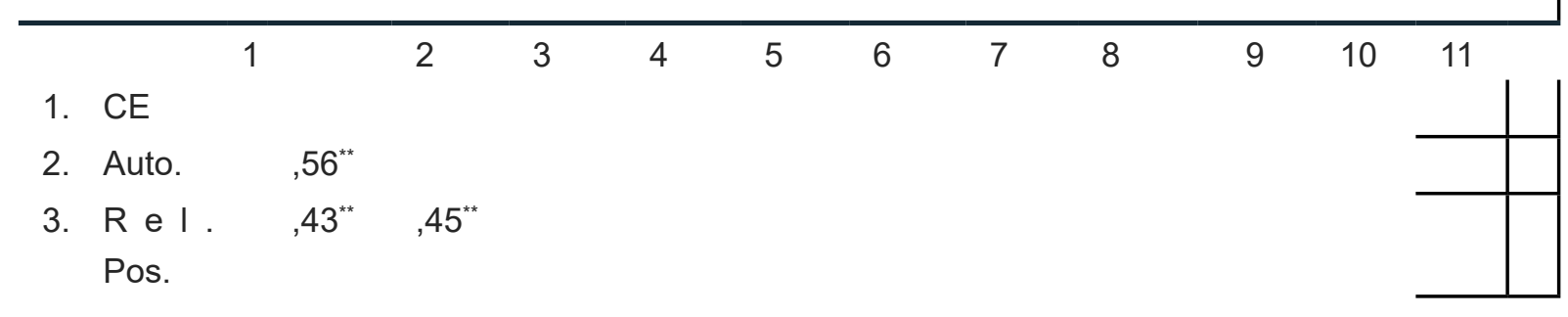


4. Aut. $\quad 37^{* *} \quad 47^{* *} \quad 38^{* *}$

5. D. Ent. $, 54^{* *}, 70^{* *}$ $, 52^{* *} \quad, 56^{* *}$

6. Pro. , $53^{* *}$ , $80^{* *}$ $, 48^{* *}, 43^{* *}$ Vid.

7. Cre $, 54^{* *} \quad, 51^{* *}$ , $42^{* *}$ , $38^{* *}$ Per.

8. Sobre. $-, 11 \quad-, 02$

, 02

9. Aser. , $07 \quad-, 01$

,06

$-, 20^{* *}$ $-, 14^{*} \quad-, 06$

, 01

$$
, 02
$$

$-, 14^{*}, 01$
$-, 08$

10. Inhi.

$-, 07 \quad-, 09$

$-, 09$

, 02

11. Puni.

$-, 09$

$-, 10 \quad, 08$

$-, 11 \quad-, 09$

$-, 08 \quad, 26^{* *}$

$\begin{array}{llll}-, 06 & -, 12 & -, 13 & , 02\end{array}$

, 09
, $38^{* *}$

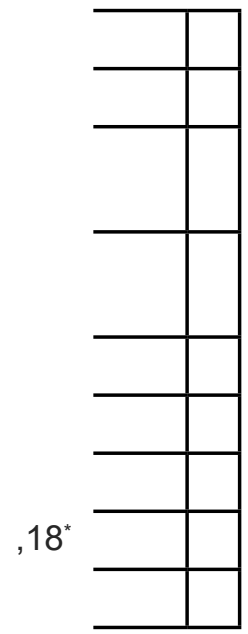

CE.Competencia emocional (PEC); F.Auto.: Factor autoaceptación (PBP); F. Rel. Pos.: Factor relaciones positivas (PBP); F. Aut.: Factor autonomía (PBP); F. Dom. Ent.: Factor dominio del entorno (PBP); F. Prop. Vid: Factor Propósito de vida (PBP); F. Crec. Per: Factor crecimiento personal (PBP); Sobre.: Total sobreprotector (PEE); Aser.: Total asertivo (PEE); Inhi.: Total inhibicionista (PEE); y Puni.: Total punitivo (PEE).

**. La correlación es significativa en el nivel 0,01 (bilateral). *. La correlación es significativa en el nivel 0,05 (bilateral).

\section{Discusión}

El momento que se está viviendo en la actualidad, es cada vez más exigente con los alumnos, profesores y familias. Se pide una capacidad de adaptación inmediata, una respuesta eficaz a estímulos diferentes y un saber estar definido como un dominio del entorno, a nivel interpersonal e intrapersonal. Esto hace que el nivel de estrés y de no control este en aumento, sobre todo para aquellas personas que trabajan en ámbito social.

Es un hecho que los docentes deben enseñar educación emocional a sus alumnos, pero debemos cuidar esa formación previa en ellos, ya que son modelo a imitar.
Los docentes evaluados en este estudio muestran que una mayor competencia emocional conlleva más auto aceptación, relaciones positivas, autonomía, dominio del entorno, propósito de vida y crecimiento personal. Pérez-Escoda y Alegre (2018) y Extremera y Rey (2016) mostraron la relación entre las competencias emocionales y bienestar.

Asimismo, a mayor competencia emocional menos inhibición, entendida como castigos aleatorios, pocos elogios, pocas situaciones controladas de aprendizaje (Magaz, y GarcíaPérez, 2011).

Interpretar mejor las emociones (altas puntuaciones en competencia emocional) supone poseer más recursos para enfrentarse a los comportamientos negativos del entorno y una menor insatisfacción de las experiencias negativas disfrutando más de las positivas (López-Cassá, Pérez-Escoda y Alegre, 2018).

El profesorado emocionalmente competente está mejor preparado para relacionarse de manera positiva y adecuada con la comunidad educativa, aumentando la eficiencia de la educación (Bisquerra, 2009) y se subraya el papel de las competencias emocionales (Ros, Filella, Riber y Pérez-Escoda, 2017) sobre el clima de aula, rendimiento académico y la autoestima. 
Se confirma la hipótesis que, a mayor competencia emocional, mayor bienestar.

Una alta competencia emocional influirá directamente en el bienestar del docente, proporcionando en éste una mayor implicación, un mayor entusiasmo en su labor docente y un perfil asertivo y de liderazgo escolar exitoso, lo cual posibilitará, indudablemente, una mejora de nuestro sistema escolar (Hernández-Castilla, Murillo e Hidalgo, 2017).

Para los profesionales de la educación es una necesidad fundamental tener un alto nivel en competencias emocionales, otras profesiones, que no implican un trato personal de tantas horas con un número tan elevado de personas, requieren un perfil diferente en esas competencias (Albaladejo, 2017)

En conclusión tal y como señala Carpena (2017), una mayor felicidad e implicación en su trabajo se destacará en las escuelas donde la actitud positiva es la norma.

\section{REFERENCIAS BIBLIOGRÁFICAS}

Bisquerra R. (2003). Educación emocional y competencias básicas para la vida. Revista de Investigación Educativa 21(1), 7-43.

Bisquerra R. (2005).La educación emocional en la formación del profesorado. Revista Interuniversitaria de Formación del Profesorado 19(3), 95-114.

Bisquerra R. (2009). Psicopedagogia de las emociones. Revista de la Asociación de Inspectores de Educación de España 16.

Bisquerra R. y Hernández N. (2017). Psicología positiva, educación emocional y

el programa aulas felices. Papeles del Psicólogo I Psychologist Papers, 38(1), 58-65.

Bisquerra R., y Pérez N. (2009). Las competencias emocionales. Educación XXI 10, 61-82.

Bisquerra R. (2013). Cuestiones sobre el Bienestar. Madrid. Síntesis.

Carpena, A. (2003). Educación socioemocional en la etapa de primaria. Editorial Octaedro. Barcelona.

Carter, D. (2016).A Nature-Based SocialEmotional Approach to Supporting

Young Children's Holistic Development in Classrooms With and Without Walls:

The Social-Emotional and Environmental Education Development (SEED) Framework. The International Journal of Early Childhood Environmental Education, 4(1), 9-24.

Cava, M.J. (2003). Comunicación familiar y bienestar psicosocial en adolescentes. En L. Gómez Jacinto (coord.), Encuentros en Psicología Social (1), 23-27

Cazalla N., y Molero D. (2014). Inteligencia emocional percibida, ansiedad $y$ afectos en estudiantes universitarios. Revista Española de Orientación y Psicopedagogía 25(3), 56-73.

Córdoba M.E. (2017). Diseño curricular basado en competencias en la educación superior. Cuaderno de pedagogía universitaria 14(27), 6-11.

Dare, P (2017). La formación emocional del profesorado. Editorial Octaedro. Barcelona.

Diener, E. (2000). Subjective well-being: The science of happiness and a proposal for a national index. American Psychologist, 55(1), 34-43

Diener, E., Suh, E. M., Lucas, R. E., y Smith, 
H. L. (1999). Subjective well-being: Three decades of progress. Psychological Bulletin, 125, 276-302.

Dolev, N.,y Leshemb S. (2016). Teachers' emotional intelligence: The impact of training. The International Journal of Education Emotional. Special Issue Volume 8 (1), 76-94.

Extremera N., y Fernández P. (2003). La inteligencia emocional en el contexto educativo: hallazgos científicos de sus efectos en el aula. Revista de Educación $332,97-116$.

Extremera N., y Fernández P. (2004a). El papel de la inteligencia emocional en el alumnado: evidencias empíricas. Revista Electrónica de Investigación Educativa 6(2).

Extremera N., y Fernández P. (2004b). Inteligencia emocional, calidad de las relaciones interpersonales y empatía en estudiantes universitarios. Clínica y Salud 15(2), 117-37.

Fernández-Abascal, E.G., Jiménez, P. y Martín, D. (2003). Emoción y Motivación. Madrid. Editorial Centro de Estudios Ramón Areces.

Fernández, A., y López, M. (2007). La inclusión del componente emocional en la formación inicial de maestros. Una experiencia para el desarrollo de la conciencia sensorial. Revista Iberoamericana de Educación, 43, 3-25.

Ferragut M. y Fierro A. (2012). Inteligencia emocional, bienestar personal y rendimiento académico en preadolescentes. Revista Latinoamericana de Psicología 44(3), 95104.
Fragoso-LuzuriagA R. (2015). Inteligencia emocional y competencias emocionales en educación superior, ¿un mismo concepto? Revista Iberoamericana de Educación Superior VI, 16, 110-25.

Furr, R., \& Funder, D. (1998). A multimodal analysis of personal negativity.Journal of Personality andSocial Psychology,74(6), 1580-1591

Gaeta, M.L. Y López, C. (2013). Competencias Emocionales y rendimiento académico en estudiantes universitarios. Revista Electrónica Interuniversitaria de Formación del Profesorado, 16 (2), 13-25.

Gallego D.J. y Gallego, M.J. (2004). Educar la Inteligencia Emocional en el aula. PPC. Madrid.

García-Jiménez, M.L. (2017). Bienestar Emocional en Educación: Empecemos por los Maestros. (Tesis doctoral). Recuperado http://bit.ly/2oJo2CP. Universidad de Murcia

Gendrón, B., Kouremenou, E., y Rusu C. (2016). Emotional Capital Development, Positive Psychology and Mindful Teaching: Which Links?. The International Journal of Emotional Education. Special Issue Volume 8 (1), 63-74.

Gordillo Gordillo M. (2015). Análisis de la competencia emocional de los futuros docentes extremeños. (Tesis doctoral). Recuperado http://bit.ly/2Fa1GnU. Universidad de Extremadura.

Freire Rodríguez, C. (2014)El bienestar psicológico en los estudiantes universitarios: Operativización del constructo y análisis de su relación con las estrategias de afrontamiento. (Tesis doctoral). Recuperado http://bit. ly/2oOsv67. Universidad de A. Coruña 
Hernández-Castilla, R., Murillo, F .J. y Hidalgo, N. (2017). Lecciones aprendidas del estudio del liderazgo escolar exitoso. Los casos de España en el proyecto internacional ISSPP. Revista de Investigación Educativa, 35(2), 499-518

Hue, C.(2013). Una experiencia de formación en competencias emocionales del profesorado universitario. Revista d'Innovació Docent Universitària 5, 42-61

Huebner, E. S. (1991a). Initial development of the Students' Life Satisfaction Scale. School Psychology International, 12, 231-243.

Huebner, E. S. (1991b). Further validation of the students' life satisfaction scale: The independence of satisfaction and affect ratings. Journal of Psychoeducational Assessment 9, 363-368.

Limonero J., Tomás-Sábado, Fernández-Castro J., Gómez-Romero J. y Ardilla-Herrero M.J. (2012). Estrategias de afrontamiento resilientes y regulación emocional: predictores de satisfacción con la vida. Behavioral Psychology / Psicología Conductual, 20(1),183-196.

López-Cassá, E.; Pérez-Escoda, N. y Alegre, A. (2018). Competencia emocional, satisfacción en contextos específicos y satisfacción con la vida en la adolescencia. Revista de Investigación Educativa, 36(1), 57-73.

López-Goñi I. , y Goñi-Zabala J.M. (2012). La competencia emocional en los currículos de formación inicial de los docentes. Un estudio comparativo. Revista de educación 357, 467-89.

Magaz, A. y García-Pérez, E.M. (2011). Perfil de estilos educativos: sobreprotector, inhibicionista, punitivo y asertivo. Manual de referencia. Ed. COHS. Bizkaia.

Martínez-Antón et al. (2007). La satisfacción con la vida en la adolescencia y su relación con la autoestima y el ajuste escolar. Anuario de Psicología 38(2).

Martínez, M, y PertegaL-Felices, M.L. (2011). Competencias socioemocionales en el desarrollo profesional del maestro. Educación XXI 14(2), 237-60.

McCullough G., Huebner E. y Laughlin J. (2000). Life events, self-concept, and adolescents' positive subjective well-being. Psychology in the Schools. DOI:10.1002/ (SICI)1520-6807(200005)37:3<281::AIDPITS8>3.0.CO;2-2

Moreno-Murcia, J.A. y Silveira,Y. (2015). Perfiles motivacionales de estudiantes universitarios. Procesos de estudio y satisfacción con la vida. Revista Electrónica Interuniversitaria de Formación del Profesorado, 18 (3), 169181.

Murillo, F.J. y Hernández-Castilla, R. (2014). Liderando escuelas justas para la Justicia Social. Revista Internacional de Educación para la Justicia Social, 3(2), 13-32

Murillo, F.J y Krichesky, G.J. (2012). El proceso del cambio escolar. Uma guía para impulsar y sostener la mejora de la escuela. Revista Iberoamericana sobre Calidad, Eficacia y Cambio en Educación. 10. (1). 27-43

Muñoz M. (2005). Prevención del estrés psicosocial del profesorado mediante el desarrollo de competencias emocionales: el programa P.E.C.E.R.A. Revista Interuniversitaria de Formación del Profesorado 19(3), 15-36. 
Palomero P., Fernández R., y Brackett M.A. (2008). La inteligencia emocional como una competencia básica en la formación inicial de los docentes: algunas evidencias. Electronic Journal of Research in Educational Psychology 6(15), 437-54.

Palomero P. (2009). Desarrollo de la competencia social y emocional del profesorado: una aproximación desde la psicología humanista. Revista Interuniversitaria de Formación del Profesorado 12(2), 145-53.

Peñalva A., López-Goñi J., y Landa N. (2012). Competencias emocionales del alumnado de magisterio: posibles implicaciones profesionales. Revista de Educación 362, 690-712.

Pérez N., Soldevila A., y Fondevila A. (2013). Evaluación de un programa de educación emocional para profesorado de primaria. Educación XXI 16(1), 233-53.

Peterson,E. R., Farruggia, S. F., Hamilton,R. J. y Brown, G. T. L. (2013).Socio-emotional key competencies; can the be mesaured and what do they relate to? Teachers and Curriculum 13, 33-45

Repetto, E., Pérez J.C. (2007). Formación en competencias socioemocionales a través de las prácticas en empresas. Revista de Formación Profesional 40, 92-112.

Ryff, C. (1989a). Beyond Ponce de Leon and life satisfaction: New directions in quest of successful aging. International Journal of Behavioral Development, 12, 35-55.

Ryff, C. (1989b). Happiness is everything, or is it? Explorations on the meaning of psychological well-being. Journal of Personality and Social Psychology, 57, 1069-1081.
Ryff, C., y Singer, B. (2002). From social structure to biology. En C. Snyder y A. López (Eds.), Handbook of positive psychology (pp. 6373). Londres: Oxford University Press.

Ros A., Filella G. , Riber R. y Pérez-Escoda N. (2017).Análisis de la relación entre competencias emocionales, autoestima, clima de aula, rendimiento académico y nivel de bienestar en educación primaria. REOP. 28(1), 8 - 18.

Saarni, C. (1999). The development of emotional competence. Nueva York: Guilford.

Saarni, C. (2000). Emotional Competence. A Developmental Perspective. En BarOn, R. y J. D. A. Parker (Eds.), The Handbook of Emotional Intelligence. Theory, Development, Assessment, and Application at Home, School, and in the Workplac. San Francisco, Ca: JosseyBass, 68-91.

Sala J. (2002). Ideas previas sobre la docencia y competencias emocionales en estudiantes de CC.. de la Educación. Revista Española de Pedagogía año LX $\mathrm{n}^{\circ} 223,543-58$.

Sanjuán P. y Ávila M. (2016). Afrontamiento y motivación como predictores del bienestar

subjetivo y psicológico. Revista de Psicopatología y Psicología Clínica, 21, 1-10.

Seiz, J., Vossb, T. y Kuntera, M. (2015). When Knowing is Not Enough - the Relevance of Teachers'Cognitive and Emotional Resources for Classroom Management. Frontline Learning Research.3(1), 55 - 77.

Talvio, M. et al. (2013). Una nueva mirada a la formación en eficacia docente de Gordon (TET): Un estudio e intervención en el aprendizaje social y emocional del profesorado. Electronic Journal os 
research in educational psychology. 11(3), 693-716.

Teruel M. P. (2000). La inteligencia emocional en el currículo de la formación inicial de los maestros. Revista Interuniversitaria de Formación del Profesorado 38, 141-52.

Thompson, S. C., Sobolew-Shubin, A., Galbraith, M. E., Schwankovsky, L., y Cruzen, D. (1993). Maintaining perceptions of control: Finding perceived control in low-control circumstances. Journal of Personality and Social Psychology, 64(2), 293-304

Torrente C., Nathanson, L., Rivers, S., y Brackett, M. (2015). SREE Conference Abstract Template.

Torrijos Fincias, P. (2016). Desarrollo y evaluación de competencias emocionales para profesores mediante una intervención por programas. (Tesis doctoral). Recuperado http://bit.ly/2tcThdZ. Universidad de Salamanca

Twyman, J. y Redding, S. (2015). Personal Competencies/Personalized Learning Reflection on Instruction. Council of Chief State School Officers, Washington, DC.

Villena, M.D, Justicia, F., Fernández, E. (2016). El papel de la asertividad docente en el desarrollo de la competencia social en su alumnado. Electronic Journal os research in educational psychology. 14(39), 310332.

Yildizbas, F. (2017). The Relationship between Teacher Candidates' Emotional Intelligence Level, Leadership Styles and Their Academic Success. Eurasian Journal of Educational Research 67, 215231.

Yuste Díaz, L. (2015). Creencias de autoeficacia docente en estudiantes de magisterio:
Análisis de su relación con variables de personalidad y bienestar psicológico $y$ estudio del cambio. (Tesis doctoral). Recuperado http://bit.ly/2F8HPWd. Universidad de Valencia. 